\title{
Thyroid Related Quality of Life in Elderly with Subclinical Hypothyroidism and Improvement on Levothyroxine is Distinct from that in Young Patients (TSAGE)
}

Authors

Stefanie Recker ${ }^{1}$, Richard Voigtländer ${ }^{1}$, Anja Viehmann ${ }^{1}$, Karin Dunschen ${ }^{1}$, Helena Kerp ${ }^{1}$, Karin Frank-Raue ${ }^{2}$, Gudrun Leidig-Bruckner ${ }^{2}$, Dieter Graf ${ }^{3}$, Sebastian Lederbogen ${ }^{4}$, Johannes W. Dietrich ${ }^{5}$, Rainer Görges ${ }^{6}$, Georg Brabant ${ }^{7}$, Uwe Völker ${ }^{8}$, Torquil Watt ${ }^{9}$, Denise Zwanziger $^{1}$, Lars Christian Moeller ${ }^{1}$, Dagmar Führer ${ }^{1}$

\section{Affiliations}

1 Department of Endocrinology, Diabetes and Metabolism, Clinical Chemistry - Division of Laboratory Research, University Hospital Essen, University Duisburg-Essen, Essen, Germany

2 Endocrine Practice, Molecular Laboratory, Heidelberg, Germany

3 Endocrine and Nuclear Medicine Center Lüneburg, Lüneburg, Germany

4 Private Endocrine Practice, Essen, Germany

5 Endocrinology and Diabetes Department, Medical Hospital I, Bergmannsheil University Hospitals, Ruhr University Bochum, Bochum, Germany

6 Department of Nuclear Medicine, Medical Faculty, University Duisburg-Essen, Essen, Germany

7 Experimental and Clinical Endocrinology, Medical Clinic I, University of Luebeck, Luebeck, Germany

8 Institute for Genetics and Functional Genome Research, University of Greifswald, Greifswald, Germany

9 Department of Medical Endocrinology, Copenhagen University Hospital Rigshospitalet, Copenhagen, Denmark

Key words

subclinical hypothyroidism, quality of life, aging, elderly, thyroid-stimulating hormone, levothyroxine treatment

received 28.01.2019

accepted 16.04.2019

Bibliography

DOI https://doi.org/10.1055/a-0897-8785

Horm Metab Res 2019; 51: 568-574

(c) Georg Thieme Verlag KG Stuttgart · New York

ISSN 0018-5043

Correspondence

Prof. Dr. Dr. med. Dagmar Führer

Department of Endocrinology, Diabetes and Metabolism,

University Hospital Essen,

University Duisburg-Essen,

45122 Essen, Germany

Tel.: + 49/201/723 6401, Fax: + 49/201/723 5972

dagmar.fuehrer@uk-essen.de
Supplementary Material for this article is available online at http://www.thieme-connect.de/products.

\section{ABSTRACT}

The aim of this study was to investigate in a longitudinal approach whether levothyroxine (LT4) substitution has a different impact on quality of life (QoL) and thyroid related QoL in younger ( $<40$ years) and older subjects ( $>60$ years) with elevated thyroid-stimulating hormone (TSH) concentrations. The study included male and female patients with newly diagnosed, untreated subclinical hypothyroidism defined by TSH $>8 \mathrm{mU} / \mathrm{l}$. Patients were recruited throughout Germany from 2013-2016 and evaluated by clinical assessment, blood sampling and questionnaires for health related QoL and thyroid-disease thyroid-related QoL (ThyPRO) at time of diagnosis and six months after initiation of LT4 treatment. We found significantly lower QoL in both young and old patients with subclinical hypothyroidism compared to age-matched healthy individuals. Higher scores on follow-up were found in all patients irrespective of age, indicating better QoL on LT4 therapy. Analysis of the ThyPRO questionnaire showed that old patients experienced less Emotional Susceptibility, Tiredness, and Impaired Day Life on LT4, while young patients reported less Cognitive Complaints, Emotional Susceptibility, and Impaired Day Life compared to baseline assessment. Hypothyroidism with TSH concentrations $>8 \mathrm{mU} / \mathrm{l}$ is associated with impairment in general and ThyPRO QoL in young and old age. Older patients benefited from LT4 therapy and remarkably show similar degree of improvement as younger patients, albeit with some thematic variation in ThyPRO QoL. Our data confirm current recommendations on initiation of LT4 substitution and suggest that this should not be withheld in elderly with TSH concentration above $8-10 \mathrm{mU} / \mathrm{l}$. 

ABBREVIATIONS
SHT Subclinical hypothyroidism
LT4 Levothyroxine
fT3 Free 3',3,5-trioodothyronine
fT4 Free thyroxine
TSH Thyroid stimulating hormone
QoL Quality of life
HRQL Health related quality of life

\section{Introduction}

Thyroid dysfunction, in addition to its negative effects on health, may severely impair well-being and quality of life $(\mathrm{QoL})$. While there is agreement that overt hyper- and hypothyroidism require treatment, the situation is less clear for subclinical hypothyroidism (sHT), defined as a thyroid-stimulating hormone (TSH) level above laboratory references range while free thyroxine (fT4) concentration is still within the normal range $[1,2]$. In this situation, where elevated TSH values are understood as a continuum [1], additional criteria may be needed for the decision to start levothyroxine (LT4) substitution. Furthermore, sHT does not necessarily progress to overt hypothyroidism and the chance of a spontaneous remission of elevated TSH is comparatively high with TSH concentrations at the upper reference range [3].

For older patients the situation is even more complex. Large epidemiological cohorts such as NHANES, TEARS, or Busselton Health Study showed that TSH concentrations rise with age, which appears not to be due to an increased incidence of autoimmune thyroid disease, but rather a shift of the TSH range with age (e. g., Busselton Health, NHANES) [4-6]. Hence, in their current guidelines the European Thyroid Association (ETA) and the American Thyroid Association (ATA) recommend treatment of hypothyroidism in elderly patients (> 70 years) only if the TSH value is increased $>8-10 \mathrm{mU} / \mathrm{I}$ (over six months) and symptoms are present $[1,7]$.

In addition, hypothyroidism is often oligosymptomatic in the elderly. Thus the question arises whether there is a specific decrease in QoL associated with hypothyroidism, in addition to the expected decrease with aging. One of the main goals of treatment of chronic disease is to improve patients' symptoms, to enable them to participate in everyday life and to keep them living in the community. For elderly patients, higher QoL may even have a greater significance than longevity [8]. The importance of QoL has also increasingly been acknowledged for thyroid disorders by some recent studies $[9,10]$. QoL is reduced in younger patients with overt hypothyroidism and improves on LT4 [11-14]. So far, no study has investigated QoL in elderly patients with overt hypothyroidism and only few studies have examined QoL in elderly patients with sHT with inconsistent findings. For example, Jaeschke and colleagues [12] did not observe a benefit of LT4 treatment in a cohort of middle-aged or elderly patients (i. e., older than 55 years) with $\mathrm{SHT}$ in a randomized placebo-controlled study. Furthermore, a recent prospective randomized placebo-controlled trial showed no apparent benefits of treatment of elderly patients with mild sHT [15] ( Table 1). However, other data suggest that overt hypothyroidism as well as sHT may represent a serious risk factor for depression and anxiety in middle-aged and elderly patients $[16,17]$.
Thus, age and QoL could be additional parameters to determine when and if LT4 substitution is indicated. Here we report results of the prospective, longitudinal Thyroid Study of Age in Germany (TSAGE), which investigates QoL in older ( $>60$ years) and younger (<40 years) patients with new onset of sHT defined as TSH concentrations above $8 \mathrm{mU} / \mathrm{I}$ at baseline and 6 months after initiation of LT4 substitution. We used a generic (SF-36) and a disease-specific (ThyPRO) questionnaire on QoL to obtain valid health related QoL (HRQL) and to maximize precision and sensitivity. Additionally, the longitudinal approach allowed us to examine possible age-differences in overall and thyroid disease related QoL on LT4 substitution between old and young patients after restoration of euthyroidism.

\section{Ethical approval}

All procedures performed in studies involving human participants were in accordance with the ethical standards of the institutional and/or national research committee and with the 1964 Helsinki declaration and its later amendments or comparable ethical standards. Informed consent was obtained from all individual participants included in the study.

\section{Patients and Methods}

\section{Patient populations}

Thyroid Study of Age in Germany (TSAGE): The study included male and female patients older than 18 years with newly diagnosed, untreated, overt endogenous hypothyroidism (TSH $>8 \mathrm{mU} / \mathrm{l}$, fT4 $<11.5 \mathrm{pmol} / \mathrm{l}$ and/or fT3 $<3.5 \mathrm{pmol} / \mathrm{I}$ ) or subclinical hypothyroidism (TSH $>8 \mathrm{mU} / \mathrm{l} ; \mathrm{fT} 4$ and $\mathrm{fT} 3$ within the reference range). Patients were recruited in six endocrine practices throughout Germany. The recruitment period was 4 years (01.01.2013-31.12.2016). Patients were evaluated by clinical assessment, blood sampling and questionnaires to measure health related QoL (SF-36 Version 2) and thyroid-disease thyroid-related QoL (ThyPRO). Evaluation took place at time of diagnosis (T1) and 6 months after initiation of LT4 treatment (T2).

Only patients with confirmed hypothyroidism with TSH $>8 \mathrm{mU} / \mathrm{l}$ (second laboratory analysis from aliquot sample) at baseline and only patients who were confirmed euthyroid after at least 6 months duration of LT4 substitution were entered in the present analysis. Clinical variables including age, cause and symptoms of hypothyroidism, blood pressure, heart rate, body weight, history of smoking were collected during the survey. Written informed consent of patients who agreed to participate in the study was taken and ethics permission was granted (Nr. 12-5132-BO).

\section{Quality of life measures}

QoL was assessed using the German version 2 of the SF-36 and the ThyPRO. The SF-36 Health Survey consists of eight subscales: Physical Function (PF), Social Functioning (SF), Role Limitations due to Physical (RP) or Emotional (RE) Problems, Mental Health (MH), Vitality (VT), Bodily Pain (BP), and General Health $(\mathrm{GH})$, with scores ranging from 0 (low) to 100 (high QoL level). Two component summary measures of SF-36, that is, Physical Component Summary (PCS) and Mental Component Summary (MCS), can be calculated too. They are standardized to a mean score of 0 and a standard deviation of 100 with high- 
- Table 1 Studies on age impact on change of hypothyroid symptoms under levothyroxine substitution.

\begin{tabular}{|c|c|c|c|c|c|c|c|c|}
\hline & \multicolumn{2}{|c|}{ Winther et al. (2016) } & \multicolumn{2}{|c|}{ TRUST; Stott et al. (2017) } & \multicolumn{2}{|l|}{ TSAGE } & \multicolumn{2}{|c|}{ Jaeschke et al. (1996) } \\
\hline & $\begin{array}{l}\text { General } \\
\text { Population }\end{array}$ & AIT & Placebo & LT4 & $\begin{array}{l}\text { Young patients } \\
\text { ( }<40 \text { years) AIT }\end{array}$ & $\begin{array}{l}\text { Old patients } \\
\text { (>60 years) AIT }\end{array}$ & Placebo & LT4 \\
\hline $\mathrm{n}$ & 739 & 78 & 369 & 368 & 17 & 11 & 18 & 19 \\
\hline Age & $50(18-81)$ & $47(18-91)$ & $74 \pm 6.8$ & $74 \pm 5.8$ & $27 \pm 6.4$ & $66 \pm 6.4$ & $68 \pm 9.4$ & $68 \pm 6.4$ \\
\hline TSH mU/l baseline & $<4.0$ & $8.1(5.5-12.2)$ & $6.4 \pm 2$ & $6.4 \pm 2$ & $15.7 \pm 7.9$ & $12.6 \pm 8.5$ & $9.4 \pm 3.1$ & $12.3 \pm 6.8$ \\
\hline Tiredness * & $35 \pm 16$ & $58 \pm 24$ & $25.5 \pm 20.3$ & $25.9 \pm 20.6$ & $57 \pm 10$ & $62 \pm 8$ & n.d. & n.d. \\
\hline Hypothyroid symptoms * & $14 \pm 21$ & $27 \pm 28$ & $16.9 \pm 17.9$ & $17.5 \pm 18.8$ & $29 \pm 10$ & $37 \pm 20$ & n.d & n.d. \\
\hline
\end{tabular}

AIT: Autoimmune thyroiditis; $\mathrm{n}=$ sample size. Age (mean/median); TSH mU/I (median, mean TSH; * ThyPRO Scales Tiredness and Hypothyrodism \pm standard deviation or range. n.d.: Not determined.)

er scores indicating better QoL. In addition to the subscales, one single-item measures the change in health status during the last year.

ThyPRO is a disease-specific QoL questionnaire developed by T. Watt $[10,18,19]$ for evaluating the QoL of patients with benign thyroid diseases. It is a robust standardized questionnaire that has been translated and validated in several languages, including German. The questionnaire consists of 84 items summarized in 13 scales (Goiter Symptoms, Hyperthyroid Physical Symptoms, Hypothyroid Physical Symptoms, Eye Symptoms, Tiredness, Cognitive Impairment, Anxiety, Depressivity, Emotional Susceptibility, Impaired Social Life, Impaired Daily Life, Impaired Sex Life and Cosmetic Complaints) and one overall QoL item. Each of the 13 ThyPRO scales is scored as a summary score and linearly transformed to a range of $0-100$, with higher scores indicating more symptoms or impairment.

\section{Laboratory parameters}

TSH, fT3 and fT4 concentrations of all patients in this study were initially measured in the private practices at T1 and T2. A second independent analysis was performed in Clinical Chemistry - Division of Laboratory Research at the University Hospital Essen. Prior to analysis in Essen, serum samples were stored at $-80{ }^{\circ} \mathrm{C}$ and accurate temperature was controlled by an in-house master display in the West German Biobank at the University Hospital Essen.

Thyroid function parameters (TSH, fT3, and fT4) were determined on thawed serum samples with the Siemens ADVIA Centaur immunoassay-system (Siemens Healthineers, Erlangen, Germany). Reference ranges, according to the manufacturer, were: $\mathrm{TSH}=0.3-$ $3.0 \mathrm{mU} / \mathrm{l}$, fT3 $=3.5-6.5 \mathrm{pmol} / \mathrm{l}$ and fT4 $=11.5-22.7 \mathrm{pmol} / \mathrm{l}$. The ADVIA Centaur ${ }^{\circledR}$ TSH3-Ultra Assay is a chemiluminescence immunoassay with intra-assay variation $<4.3 \%$, inter-assay variation $<4.7 \%$, functional sensitivity of $0.008 \mathrm{mU} / \mathrm{l}$ and detection limit at $0.008 \mathrm{mU} / \mathrm{l}$. The ADVIA Centaur ${ }^{\circledR}$ fT3- and fT4-Assays are competitive chemiluminescence immunoassays with intra-assay variations $<4.1 \%$ for fT 3 and $<4.0 \%$ for fT 4 , inter-assay variations $<3.1 \%$ for fT 3 and $<3.3 \%$ for fT 4 and analytical sensitivity of $0.3 \mathrm{pmol} / \mathrm{l}$ for fT3 and $1.3 \mathrm{pmol} / \mathrm{I}$ for fT4, respectively. The Department of Clinical Chemistry is accredited according to DIN EN ISO 15189:2014.

\section{Statistical analyses}

The change to treatment in health related QoL was evaluated as the change in scale scores in the SF-36 and the ThyPRO from baseline (T1) to follow-up (T2).

In order to take age- and sex-dependent differences in generic QoL into account, the final SF-36 scores were corrected for age- and sex- specific values from the German norm-base dataset. The German version of the SF-36 was used, for example, in the Federal Health Survey 1998 and was standardized on a sample of 6964 people between ages of 18 to 80 years [20].

The differences between patients' values and the age- and sexspecific normative values was calculated for $\mathrm{T} 1$ and $\mathrm{T} 2$ separately. To evaluate the response to treatment, $\mathrm{T} 2$ values and $\mathrm{T} 1$ values were subtracted. In order to examine whether the effect of therapy on health related QoL differed between older patients ( $>60$ years of age) and younger patients (18-40 years of age) an independent sample t-test for these differences was carried out.

For the evaluation of the ThyPRO, changes in mean scale scores were compared between T2 and T1 using the paired Student's ttest. Values of $\mathrm{p}<0.05$ were considered statistically significant.

In addition to statistical significance testing, effect sizes (mean difference divided by standard deviation at baseline) were also calculated, to enhance interpretability [13]. In accordance with Cohen [21] effect sizes between 0.2 and 0.5 were classified as small, $0.5-$ 0.8 as moderate, and 0.8 as large.

All analyses were performed with SPSS software, version 22.0 (Inc., Chicago, IL, USA).

\section{Results}

Fourty-six patients with newly diagnosed subclinical hypothyroidism (TSH > $8 \mathrm{mU} / \mathrm{l})$, fT4, and fT3 within range ( $\mathrm{fT} 4=11.5-22.7 \mathrm{pmol} / \mathrm{l}$; fT3 $=3.5-6.5 \mathrm{pmol} / \mathrm{l}$ ) completed the ThyPRO and SF-36 (Version 2) at baseline (T1). Twenty eight patients ( $>60$ years $n=11 ;<40$ years $\mathrm{n}=17$ ) completed the study at 6 months after treatment initiation (T2) and all were euthyroid at T2. In the remaining 18 patients, data sets were either incomplete or patients did not reach the treatment goal (euthyroidism) at T2. 
- Table 2 Age, heart rate, mean arterial blood pressure (MAD), and thyroid function parameters in the study population before and six months after initiation of levothyroxine substitution.

\begin{tabular}{|c|c|c|c|c|}
\hline & \multicolumn{2}{|l|}{ Baseline } & \multicolumn{2}{|l|}{ Follow-up } \\
\hline & Old Patients $(n=11)$ & Young Patients $(n=17)$ & Old Patients $(n=11)$ & Young Patients $(n=17)$ \\
\hline Age & $66(60-75)$ & $26(19-35)$ & $67(61-76)$ & $27(20-36)$ \\
\hline MAD & $98(96-106)$ & $91(71-106)$ & $96(88-103)$ & $93(60-80)$ \\
\hline Heart rate & $72(60-96)$ & $73(64-88)$ & $69(60-80)$ & $70(88-113)$ \\
\hline TSH & $13(8-32)$ & $16(8-85)$ & $3(1-3)$ & $2(0-3)$ \\
\hline fT4 & $16(12-21)$ & $14(12-18)$ & $21(15-23)$ & $18(14-23)$ \\
\hline fT3 & $4(3-5)$ & $5 \pm 1(3-6)$ & $5(4-6)$ & $5(4-6)$ \\
\hline
\end{tabular}

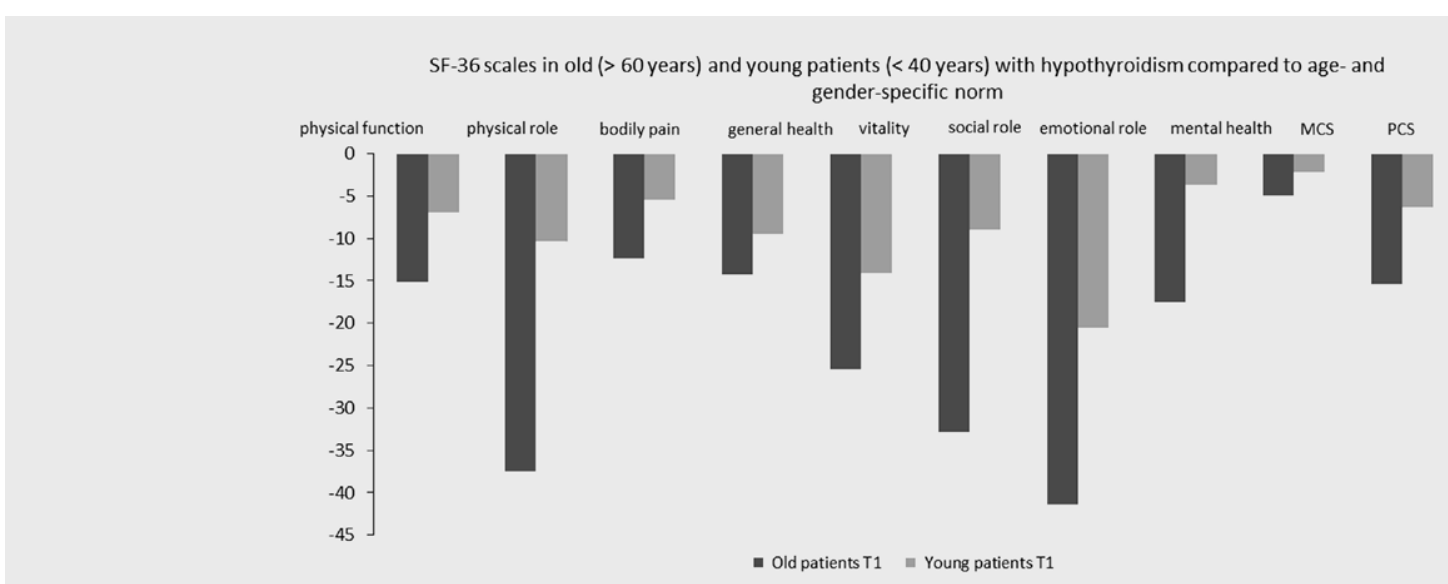

- Fig. 1 T1: newly diagnosed untreated hypothyroidism (baseline) defined as TSH $>8 \mathrm{mU} / \mathrm{l}$. Shown are differences to the respective age- and genderspecific norm in the SF-36 questionnaire.

Age-impact on QoL was evaluated in two thyroid patient groups: patients with $\mathrm{sHT}>60$ years of age ("old patients", $\mathrm{N}=11$; male: 5 ; female: 6) and 40 years of age ("young patients", $n=17$; male: 4 , female: 13). Age, mean arterial blood pressure and thyroid function tests are presented in $>$ Table $\mathbf{2}$. There was no significant difference neither in clinical parameters (heart rate, mean arterial blood pressure) nor TSH, fT 4 and fT 3 concentrations between the two patient groups.

Subclinical hypothyroidism was due to autoimmune thyroid disease (AIT) in all patients, confirmed by elevated thyroid peroxidase autoantibodies (above reference range in laboratory of private practice) and thyroid ultrasound findings suggestive of AIT (documented in private practice).

\section{Differences in QoL to the age and standard specific norm}

Generic QoL, measured with the SF-36 Health Survey, was significantly lower in both young and old patients with sHT compared to healthy individuals. Larger differences were found in all QoL sub- scales in old compared to young patients and persisted after correction for age- and gender-matched healthy individuals ( $\triangleright$ Fig. 1 and $\triangleright$ Table 3 ). Treatment with LT4 was associated with significant improvement in SF-36 subscales Physical Function and Vitality and the calculated Physical Component Summary (PCS) in old patients while young patients reported a significant improvement in SF-36 subscales Physical Function and Social Functioning and the derived Mental Component Summary (MCS) ( $\triangleright$ Table 3 ). There was no change in Mental Health from baseline to follow-up on treatment in either patient group. For all other SF-36 subscales, all patients showed higher scores on follow-up, irrespective of age, that is, better QoL on LT4 therapy.

With regard to thyroid specific QoL (ThyPRO), old patients experienced significantly less Tiredness and Impaired Day Life in addition to a significant improvement in Emotional Susceptibility under LT4 substitution $(p<0.05)$. Young patients reported significantly less Impaired Day Life, Cognitive Complaints and Emotional Susceptibility compared to baseline $(p<0.05$, $>$ Table 4$)$. 
Table 3 Impact of age and levothyroxine substitution on SF-36 scales.

\begin{tabular}{|c|c|c|c|c|c|c|c|c|}
\hline & \multicolumn{4}{|c|}{ Old patients $(n=11)$} & \multicolumn{4}{|c|}{ Young patients $(n=17)$} \\
\hline & Baseline & Follow-up & Change & p-Value & Baseline & Follow- up & Change & p-Value \\
\hline Physical function & $-15 \pm 31$ & $-11 \pm 30$ & 4 & 0.642 & $-7 \pm 14$ & $-1 \pm 10$ & 6 & 0.039 \\
\hline Physical problems * * & $-37 \pm 42$ & $-15 \pm 43$ & 22 & 0.029 & $-10 \pm 27$ & $1 \pm 18$ & 9 & 0.104 \\
\hline Body pain & $-12 \pm 40$ & $-5 \pm 42$ & 7 & 0.284 & $-5 \pm 26$ & $0 \pm 17$ & 5 & 0.411 \\
\hline General health & $-14 \pm 20$ & $-6 \pm 26$ & 8 & 0.139 & $-9 \pm 18$ & $-8 \pm 26$ & 1 & 0.686 \\
\hline Vitality & $-25 \pm 18$ & $-13 \pm 21$ & 12 & 0.036 & $-14 \pm 20$ & $-10 \pm 21$ & 4 & 0.225 \\
\hline Social Function & $-33 \pm 27$ & $-26 \pm 29$ & 7 & 0.523 & $-9 \pm 15$ & $1 \pm 12$ & 8 & 0.003 \\
\hline Emotional problems ** & $-41 \pm 43$ & $-34 \pm 50$ & 7 & 0.678 & $-20 \pm 39$ & $-6 \pm 30$ & 14 & 0.312 \\
\hline Mental health & $-18 \pm 17$ & $-18 \pm 17$ & 0 & 0.146 & $-4 \pm 15$ & $-4 \pm 15$ & 0 & 0.151 \\
\hline MCS & $-5 \pm 13$ & $-1 \pm 15$ & 4 & 0.55 & $-2 \pm 7$ & $0 \pm 5$ & 2 & 0.045 \\
\hline PCS & $-15 \pm 11$ & $-13 \pm 13$ & 2 & 0.068 & $-6 \pm 10$ & $-3 \pm 9$ & 3 & 0.216 \\
\hline
\end{tabular}

Mean differences \pm standard deviation from gender and age specific/matched norm. ${ }^{*}$; Mean deviations from the age- and gender matched normal population (baseline) and 6 months after initiation of levothyroxine substitution (follow-up). Significant changes $(p<0.05$ ) from baseline to follow up are indicated; " German norm-based dataset of the SF36; " Summary (MCS), Physical Component Summary (PCS).

The magnitudes in changes, estimated by the standardized effect sizes, at 6 months after initiation of LT4 treatment, are shown for each of the ThyPRO Scales in (Supplemental Table 1S). In old patients the change under LT4 substitution was large $(>0.8)$ for Tiredness and moderate (0.5-0.8) for Impaired Day Life, small (0.20-0.50) for Eye Symptoms, Cognitive Problems, Anxiety, Emotional Susceptibility, Social Impairment, Impaired Sex Life and Cosmetic Complaints. There were no changes $(<0.20)$ in four scales: Goiter Symptoms, Physical Hyperthyroid Symptoms, Physical Hypothyroid Symptoms and Depressivity.

In young patients, the change in Impaired Day Life was moderate under LT4 substitution (0.5-0.8), and only small changes (0.20-0.50) were found for Eye Symptoms, Cognitive Problems, Anxiety, Depressivity, Social Impairment, and Cosmetic Complaints. Six scales showed no change (<0.20): Goiter Symptoms, Physical Hyperthyroid Symptoms, Physical Hypothyroid Symptoms, Tiredness, Emotional Susceptibility, Impaired Sex Life.

Thus, compared to young patients, LT4 substitution in old patients with hypothyroidism was associated with more pronounced changes in thyroid disease specific QoL in addition to larger improvement of Tiredness from T1-T2. Conversely, Depressivity did not change in the older patient group, whereas in younger patients a small change in Depressivity was found after six months of LT4 therapy.

\section{Discussion}

In this prospective study, we assessed generic (SF-36) and disease-specific (ThyPRO) QoL with validated questionnaires in young and elderly patients with newly diagnosed, untreated sHT and analyzed the impact of age on effects of LT4 substitution on QoL. Ad- ditionally, the health related quality of life (HRLQ) was compared with age- and sex adjusted normative values from a general German population sample for the SF-36. We show that using TSH concentrations $>8 \mathrm{mU} / \mathrm{l}$ as a cut-off, old patients with untreated $\mathrm{sHT}$ benefit from LT4 substitution in terms of HRLQ and thyroid disease specific QoL, in part with even more pronounced improvement in QoL and distinct pattern of QoL compared to young patients.

Unlike Stott et al. [15], the TSAGE cohort showed an improvement in most areas of general and disease specific QoL. We found the strongest of all changes in disease related tiredness. Interestingly, the Tiredness scores (ThyPRO) in the TRUST cohort was 25.5 in the placebo and 25.9 in the LT4 group before treatment (scale from 0-100; higher scores indicating more symptoms). Compared to other populations this is quite low. For example in a Danish general population sample ( $n=739$, average age of 50 years) the baseline Tiredness score was 35 [14]. Given this discrepancy it is perhaps not surprising that Tiredness scores did not show further improvement in the TRUST cohort on LT4 treatment (28.6 in the placebo group and 28.7 in the LT4 group) [15]. In our TSAGE cohort, old subclinical hypothyroid patients reported an average Tiredness score of 62 and young patients of 57 . Other studies reported Tiredness scores of 61 in cohorts with sHT and an average age of $45[13,14]$.

Furthermore, the design of the TRUST study differed from our study approach. Although TSH concentrations above > 4.6$19.99 \mathrm{mU} / \mathrm{I}$ were defined as an inclusion criterion in TRUST, the mean TSH concentration in the treatment and placebo group was only $6.40 \pm 2.01 \mathrm{mU} / \mathrm{l}$. This was more in line with an age-adjusted normal TSH concentration in individuals $>65$ years than a pathological TSH finding. In our study, only patients with TSH-concentrations $>8 \mathrm{mU} / \mathrm{I}$ were included, which is at the threshold suggest- 
Table 4 Impact of age and levothyroxine substitution on ThyPRO items.

\begin{tabular}{|c|c|c|c|c|c|c|c|c|}
\hline & \multicolumn{4}{|c|}{ Old patients T1 $(n=11)$} & \multicolumn{4}{|c|}{ Young patients $(n=17)$} \\
\hline & Baseline & Follow-up & Change & p-Value & Baseline & Follow- up & Change & p-Value \\
\hline Goiter symptoms & $38 \pm 22$ & $36 \pm 17$ & -2 & 0.657 & $28 \pm 11$ & $28 \pm 9$ & 0 & 0.87 \\
\hline Hyperthyroid symptoms & $44 \pm 16$ & $46 \pm 16$ & +2 & 0.556 & $35 \pm 11$ & $34 \pm 9$ & -1 & 0.607 \\
\hline Hypothyroid Symptoms & $37 \pm 20$ & $35 \pm 18$ & -2 & 0.518 & $29 \pm 10$ & $29 \pm 12$ & 0 & 0.1 \\
\hline Eye symptoms & $41 \pm 20$ & $39 \pm 23$ & -2 & 0.456 & $31 \pm 10$ & $34 \pm 18$ & +3 & 0.648 \\
\hline Tiredness & $62 \pm 8$ & $54 \pm 11$ & -8 & 0.038 & $57 \pm 10$ & $59 \pm 9$ & +2 & 0.562 \\
\hline Cognitive problems & $55 \pm 26$ & $47 \pm 22$ & -8 & 0.226 & $41 \pm 20$ & $34 \pm 16$ & -7 & 0.032 \\
\hline Anxiety & $61 \pm 21$ & $56 \pm 19$ & -5 & 0.021 & $49 \pm 33$ & $37 \pm 19$ & -12 & 0.137 \\
\hline Depressivity & $57 \pm 17$ & $57 \pm 18$ & 0 & 1 & $50 \pm 11$ & $53 \pm 35$ & +3 & 0.779 \\
\hline Emotional susceptibility & $59 \pm 17$ & $55 \pm 13$ & -4 & 0.05 & $53 \pm 13$ & $50 \pm 12$ & -3 & 0.022 \\
\hline Social impairment & $45 \pm 24$ & $40 \pm 23$ & -5 & 0.452 & $27 \pm 10$ & $24 \pm 12$ & -3 & 0.492 \\
\hline Impaired day life & $55 \pm 23$ & $43 \pm 18$ & -12 & 0.013 & $36 \pm 15$ & $28 \pm 8$ & -8 & 0.01 \\
\hline Impaired sex life & $54 \pm 26$ & $50 \pm 15$ & -4 & 0.0191 & $28 \pm 19$ & $25 \pm 9$ & -3 & 0.351 \\
\hline Cosmetic complaints & $35 \pm 18$ & $33 \pm 14$ & -2 & 0.33 & $32 \pm 16$ & $27 \pm 12$ & -5 & 0.119 \\
\hline
\end{tabular}

ed by ETA and ATA for consideration of levothyroxine in individuals $>70$ years and in line with ATA's recommendation for adjustment of levothyroxine substitution in the elderly.

Another important aspect, which should be taken into consideration when evaluating studies examining QoL of thyroid patients, is the adequate selection of questionnaires. The ThyPRO questionnaire was designed to specifically display disease-related QoL in thyroid patients and thereby to incorporate the complex concept of QoL [10]. Furthermore, high quality psychometric criteria i. e., good reliability and validity have to be demonstrated for questionnaires. Our study used the well-established, high quality questionnaires SF-36 and all scales of the ThyPRO in order to investigate a broad spectrum of QoL in young and old sHT patients. Winther and colleagues [14] used the same instruments and compared their results also to a healthy population sample. However, their study was not designed for the comparison of young and old patients (the average age of their study population was 47 ) (see $>$ Table 1). Thus, there are no results concerning differences/similarities in QoL in distinct age groups before and after LT4 substitution.

TSAGE enabled us to investigate QoL before and after LT4 substitution with special regard to these distinct groups of age (young $<40$ and old $>60$ ) and since TSAGE is a prospective and longitudinal study, there was enough time for tissue equilibration of thyroid hormone after initiation of LT4 substitution. Similarly to Winther et al. [14] we found health related QoL to be impaired in hypothyroid patients and improved by LT4 substitution. Based on the TSAGE we show that this accounts for young and old patients.

One interesting and unexpected finding of our study was that sHT with TSH-concentrations $>8 \mathrm{mU} / \mathrm{I}$ had a larger impact on HRLQ in older than younger patients. This was consistent also after cor- rection for age- and gender matched HRQL of the normal population. Older patients reported significantly lower scores in all areas of HRQL than younger patients, except for Bodily Pain and Emotional Problems. On LT4 treatment the differences in HRQL between younger and older patients was reduced, again illustrating that both old and young patients with sHT benefit from LT4 substitution in terms of QoL. Noteworthy the physical dimension of QoL improved significantly in old patients, especially Physical Role Function (i. e., to accomplish things, perform activities, only do certain things) demonstrated the largest change. Furthermore, Vitality improved significantly. Other particular disease specific issues for older patients were Tiredness, Anxiety, Depressivity and Emotional Susceptibility. A remarkable effect of therapy was found in the amelioration of the disease specific Tiredness and notably less Impaired Day Life. This reflects as well in significantly higher scores in Vitality and Physical Problems (SF-36). A shortcoming of our study is the small sample size. Yet, our study is the first controlled follow-up study in which QoL of older patients with new onset, untreated sHT was compared to QoL of younger patients with sHT and healthy individuals of the same sex and age. Since we have not included a control group (for example administration of placebo instead of LT4), we cannot rule out a distortion of our data on QoL by comorbidities and/or positive/negative life events that increased the likelihood that our patients sought medical advice in the first place. Furthermore a TSH-concentration $>8 \mathrm{mU} / \mathrm{l}$ was confirmed in a second blood sample but not in a serial analysis. However a recent longitudinal study over 5 years has shown that TSH concentrations $>8 \mathrm{mU} / \mathrm{I}$ rarely reverse to normal range TSH concentrations but persist or progress to overt hypothyroidism, in contrast to TSH levels at the upper laboratory reference range [22]. 
Strengths of our study are the application of very strict inclusion criteria. We focused on defined age groups, that is, young patients below 40 years of age and old patients above 60 years of age. Furthermore we applied the presently discussed TSH cut-off $>8 \mathrm{mU} / \mathrm{I}$ $[1,2,7,23-25]$ rather than TSH $>4.99 \mathrm{mU} / \mathrm{l}$ used in many epidemiological studies [15]. Patients had to be newly diagnosed and untreated, which represented a challenging recruitment threshold. All patients had autoimmune thyroiditis, a confounder effects of the underlying pathogenesis of hypothyroidism on QoL is therefore less likely. To take into account pre-analytic and assay variations in determining TSH concentrations, we repeated the measurements of the TSH value in our laboratory. Moreover, we used established questionnaires with solid psychometric properties to measure general and disease specific aspects of QoL.

It may be argued that QoL is at least partly biased due to subjects' awareness of treatment. Yet, a study showed that LT4 treated previously hypothyroid patients blinded to their exact treatment status showed decrements in their perceived health status and mood when sHT was induced [26].

In conclusion, $\mathrm{SHT}$ at TSH concentrations $>8 \mathrm{mU} / \mathrm{l}$ is associated with severe impairment in general and disease specific QoL, in young and old age. Furthermore, our study demonstrates that older patients with autoimmune thyroiditis and TSH concentration $>8 \mathrm{mU} /$ I benefit from LT4 therapy in terms of QoL and show similar degree of improvements as younger patients. Furthermore, we see a confirmation of the present concept of TH homeostasis with regard to QoL in elderly patients, indicating that TSH concentration above 8-10 mU/I levels impact QoL and hence treatment should not be withheld in the young and the old.

\section{Funding}

This study was funded by the Deutsche Forschungsgemeinschaft (DFG) FU 356/7-1 and FU 356/10-1 to DF, and by DFG MO 1018/2-1 to LCM in the framework of SPP1629 "Thyroid Trans Act".

\section{Conflict of Interest}

The authors declare that they have no conflict of interest.

\section{References}

[1] Pearce SH, Brabant G, Duntas LH et al. ETA Guideline: Management of Subclinical Hypothyroidism. Eur Thyroid J 2013; 2: 215-228

[2] Ross DS, Burch HB, Cooper DS et al. American Thyroid Association Guidelines for Diagnosis and Management of Hyperthyroidism and Other Causes of Thyrotoxicosis. Thyroid 2016; 26: 1343-1421

[3] Verma A, Hasan T. Thyroid disorders in older people. Rev Clin Gerontol 2009; 19: 193-206

[4] Atzmon G, Barzilai N, Hollowell JG et al. Extreme longevity is associated with increased serum thyrotropin. J Clin Endocrinol Metab 2009; 94: 1251-1254

[5] Hollowell JG, Staehling NW, Flanders WD et al. Serum TSH, T4, and Thyroid Antibodies in the United States Population (1988 to 1994): National Health and Nutrition Examination Survey (NHANES III). J Clin Endocrinol Metab 2002; 87: 489-499
[6] Surks MI, Boucai L. Age- and race-based serum thyrotropin reference limits. J Clin Endocrinol Metab 2010; 95: 496-502

[7] Gharib H, Tuttle RM, Baskin H] et al. Subclinical thyroid dysfunction: a joint statement on management from the American Association of Clinical Endocrinologists, the American Thyroid Association, and the Endocrine Society. J Clin Endocrinol Metab 2005; 90: 581-585

[8] Brunner-La Rocca HP, Rickenbacher PMuzzarelli et al. End-of-life preferences of elderly patients with chronic heart failure. Eur Heart J 2012; 33: 752-759

[9] Bektas Uysal H, Ayhan M. Autoimmunity affects health-related quality of life in patients with Hashimoto's thyroiditis. Kaohsiung J Med Sci 2016; 32: 427-433

[10] Watt T, Groenvold M, Rasmussen AK et al. Quality of life in patients with benign thyroid disorders. A review. Eur J Endocrinol 2006; 154: 501-510

[11] Bianchi GP, Zaccheoni V, Solaroli E et al. Health-related quality of life in patients with thyroid disorders. A study based on Short-Form 36 and Nottingham Health Profile Questionnaires. Qual Life Res 2004; 13: 45-54

[12] Jaeschke R, Guyatt G, Gerstein $\mathrm{H}$ et al. Does treatment with I-thyroxine influence health status in milddle-aged and older adults with subclinical hypothyroidism? J Gen Intern Med 1996; 11: 744-749

[13] Watt T, Cramon P, Hegedus L et al. The thyroid-related quality of life measure ThyPRO has good responsiveness and ability to detect relevant treatment effects. J Clin Endocrinol Metab 2014; 99 : 3708-3717

[14] Winther KH, Cramon P, Watt T et al. Disease-specific as well as generic quality of life is widely impacted in autoimmune hypothyroidism and improves during the first six months of levothyroxine therapy. PLoS One 2016; 11: e0156925

[15] Stott D], Rodondi N, Kearney PM et al. Thyroid hormone therapy for older adults with subclinical hypothyroidism. N Engl J Med 2017; 376: 2534-2544

[16] Chueire VB, Romaldini JH, Ward LS. Subclinical hypothyroidism increases the risk for depression in the elderly. Arch Gerontol Geriatr 2007; 44: 21-28

[17] Larisch R, Kley K, Nikolaus S et al. Depression and anxiety in different thyroid function states. Horm Metab Res 2004; 36: 650-653

[18] Watt T, Hegedus L, Groenvold M et al. Validity and reliability of the novel thyroid-specific quality of life questionnaire, ThyPRO. Eur J Endocrinol 2010; 162: 161-167

[19] Watt T, Barbesino G, Bjorner JB et al. Cross-cultural validity of the thyroid-specific quality-of-life patient-reported outcome measure, ThyPRO. Qual Life Res 2015; 24: 769-780

[20] Bullinger M, Kirchberger I. SF-36 Fragebogen zum Gesundheitszustand. Göttingen: Hogrefe; 1998

[21] Cohen J. Statistical power analysis for the behavioral sciences. Hillsdale, NJ: Lawrence Erlbaum; 1988

[22] Rosário PW, Carvalho M, Calsolari MR. Natural history of subclinical hypothyroidism with TSH $\leq 10 \mathrm{mIU} / \mathrm{l}$ : a prospective study. Clin Endocrinol 2016; 84: 878-881

[23] Grossman A, Weiss A, Koren-Morag $N$ et al. Subclinical Thyroid Disease and Mortality in the Elderly: A Retrospective Cohort Study. Am J Med 2016; 129: 423-430

[24] Karmisholt ], Andersen S, Laurberg P. Variation in thyroid function in subclinical hypothyroidism: importance of clinical follow-up and therapy. Eur J Endocrinol 2011; 164: 317-323

[25] Rosario PW, Calsolari MR. Levothyroxine therapy in the subclinical hypothyroidism: a lifelong therapy? A long-term study. Clin Endocrinol (Oxf) 2016; 85: 819-820

[26] Samuels MH, Schuff KG, Carlson NE et al. Health status, mood, and cognition in experimentally induced subclinical hypothyroidism. J Clin Endocrinol Metab 2007; 92: 2545-2551 